FUNDACIÓN KOINONIA (F.K). Santa Ana de Coro. Venezuela.

Liliana Amalia Alegre-Alvarado

http://dx.doi.org/10.35381/r.k.v6i4.1440

\title{
Aprendizaje integrado de contenido y lengua extranjera para la enseñanza comunicativa de inglés académico
}

Integrated learning of content and foreign language for communicative teaching of academic English

\author{
Liliana Amalia Alegre-Alvarado \\ lyamali01@gmail.com \\ Universidad César Vallejo de Chimbote, Perú \\ https://orcid.org/0000-0002-2762-7562
}

Recepción: 01 de julio 2021

Revisado: 15 de agosto 2021

Aprobación: 15 de septiembre 2021

Publicación: 01 de octubre 2021 


\title{
RESUMEN
}

El presente artículo tiene el propósito presentar una revisión de la literatura que permita describir el Aprendizaje Integrado de Contenido y Lengua Extranjera (AICLE) como enfoque metodológico para el desarrollo didáctico de la enseñanza comunicativa de idiomas, en particular el inglés. Metodológicamente responde proceso de investigación teórica; por tanto, fue menester realizar una observación sistemática de la bibliografía que denota el conocimiento construido en este tenor, mediante el despliegue de los procedimientos metodológicos de rastreo, identificación, organización, sistematización, análisis e interpretación de datos e informaciones, como lógica de acción investigativa. Finalmente, el enfoque AICLE/CLIL se muestra como un paradigma metodológico fundamentado epistemológicamente en el acto comunicativo, donde el lenguaje se presenta como instrumento de la interacción, configurándose como una forma de aprendizaje más efectivo, pues se aboca a desarrollar competencias para el uso funcional y comunicativo de otro idioma como el inglés de manera contextualizada.

Descriptores: Enseñanza de idiomas; enseñanza de una segunda lengua; educación bilingüe. (Palabras tomadas del Tesauro UNESCO).

\begin{abstract}
The purpose of this article is to present a review of the literature that allows us to describe Content and Foreign Language Integrated Learning (CLIL) as a methodological approach for the didactic development of communicative language teaching, particularly English. Methodologically, it responds to the theoretical research process; Therefore, it was necessary to carry out a systematic observation of the bibliography that denotes the knowledge built in this vein, through the deployment of methodological procedures for tracking, identification, organization, systematization, analysis and interpretation of data and information, as a logic of investigative action. Finally, the CLIL / CLIL approach is shown as a methodological paradigm based epistemologically on the communicative act, where language is presented as an instrument of interaction, configuring itself as a more effective way of learning, as it focuses on developing skills for functional use and communicative of another language such as English in a contextualized way.
\end{abstract}

Descriptors: Language instruction; second language instruction; bilingual education. (Words taken from the UNESCO Thesaurus). 


\section{INTRODUCCIÓN}

El inglés, es la lengua por excelencia para la comunicación global en el mundo de los negocios internacionales, pues, es el idioma más hablado por una cuarta parte de los habitantes del planeta. Actualmente, existen más de 1.348 millones de hablantes en el mundo; donde, aproximadamente 379 son nativos, mientras que el resto la han aprendido como segundo idioma (Fernández, 2021).

Es de saber que la evolución histórica de la lengua inglesa se puede comprender en función de tres momentos como son: inglés antiguo, inglés medio e inglés moderno. En este sentido y considerando a Niño-Puello (2013), el inglés antiguo o anglosajón llega a las islas británicas, las cuales hablaban un idioma de origen Céltico, de la mano de tres tribus germanas denominadas Anglos, Sajones y Jutos, en los años 500 AC. Seguidamente y con el proceso evolutivo, el inglés medio es muestra de la influencia de las lenguas vikingas, del latín, que era la lengua de la iglesia y demás lenguas romances, a las reglas del anglosajón. Finalmente, el inglés moderno se considera que comenzó en el siglo XVI y se extiende hasta la actualidad. Esta etapa fue influenciada por la introducción de la primera máquina de imprenta; el establecimiento de normas de escritura y gramática, que conllevaron a la publicación del primer diccionario del idioma inglés y la expansión del imperio británico, proceso que permitió la incorporación, de manera directa 0 indirecta, de muchos términos de origen extranjero a la lengua anglosajona.

De allí que se puede decir que el inglés como lengua, se ha desarrollado a la par de la evolución histórica del mundo, y, aunque es originario de Inglaterra, con el pasar del tiempo ha llegado a ser el idioma primordial de otras regiones como: Canadá, Estados Unidos, Australia y la India. Presentándose hoy como un idioma internacional que no se limita a ser un medio de comunicación en la cotidianidad, sino, que también es una herramienta esencial que determina la vida humana y las relaciones en sectores importantes de la sociedad, como los ámbitos políticos, económicos, culturales, tecnológicos y científicos (Niño-Puello, 2013). 
Considerando lo planteado, se tiene que, en diez países de América Latina entre ellos: Brasil, Argentina, Colombia, Chile, Costa Rica, Ecuador, México, Panamá, Uruguay, y Perú; el aprendizaje del inglés se ha considerado como una cuestión estratégica sustancial, por lo que se han desarrollado grandes inversiones para crear programas estratégicos nacionales; identificando primero cuales son las dificultades que impiden dar oportunidad y mejorar el dominio del inglés. Pues, como como expresa Peña (2019), con la actual globalización mundial, donde países potencias como Inglaterra y Estados Unidos, los cuales ejercen gran influencia socio-cultural y dominan los procesos comerciales y financieros globales, se ha hecho transcendental aprender el inglés como segunda lengua, para usarlo en circunstancias cotidianas.

Cabe destacar que, de los países nombrados, Chile es el único que muestra que ha hecho frente con éxito a los marcos de políticas para lograr el aprendizaje del inglés como idioma fundamental; es decir, ha logrado un gran avance respecto a los estándares e indicadores de progreso estipulados. mientras que en los otros países aún existen brechas que han de cubrirse (Cronquist y Fiszbein, 2017).

En el escenario peruano, se tiene que después del golpe de Estado de 1968, el gobierno confinó la enseñanza del inglés; y es, en la década de los noventa, que el gobierno de turno firma un acuerdo con el país británico para coadyuvar a la enseñanza de idiomas en general y del inglés en particular (British Council, 2015). Sin embargo, esta situación se revertiría a partir del año 2015, cuando el gobierno peruano, considerando que las actuales demandas de la sociedad del conocimiento ponen en evidencia que este idioma es esencial como herramienta de acceso a la información y facilitación de la comunicación, el conocimiento y la valoración de otras cultura y saberes; oficializa la Política Nacional "inglés, puertas al mundo", mediante Decreto Supremo № 012-2015MINEDU (MINEDU, 2015). Asimismo, aprueba el Plan de Implementación al 2021 en la red de escuelas públicas, como principal instrumento orientador de las entidades del Estado para la enseñanza, aprendizaje y uso del Idioma inglés, según Decreto Supremo Nº07-2016-MINEDU (MINEDU, 2016). 
Aquí, cabe destacar lo expresa Education First (EF, 2021) con su último estudio en el que precisa que Perú ocupa el puesto 56 de 112 países que hablan inglés. Es decir, en líneas generales, se tiene un nivel moderado de dominio del inglés. No obstante, pese a este logro y a los esfuerzos realizados por el Gobierno peruano en esta materia, son evidentes las deficiencias en cuanto al proceso de enseñanza y aprendizaje del inglés como lengua extranjera.

Muestra de ello, lo plantean García, Vecorena y Velazco (2019), cuando enuncia que la realidad actual en esta materia no ha cambiado mucho en el tiempo, sobretodo si consideran los estudios realizados por Zierer-Wu en el año 1981, pues, es escasa la investigación científica en cuanto a la problemática de la enseñanza de idiomas. En este sentido y considerando los autores antes nombrados, se tiene que la política para la enseñanza, aprendizaje y uso del Idioma inglés ha sido poco efectiva, pues, los estudiantes egresan con escaza competencia y bajo nivel de conocimiento en inglés como lengua extranjera; pudiéndose destacar entre las múltiples causas, la falta de currículos y programas de estudios debidamente elaborado para la enseñanza del inglés, aunado a las escasas iniciativas de apoyo formativo y perfeccionamiento profesional de los especialistas en esta área (García, Vecorena y Velazco, 2019; p. 82-101).

De igual forma, lo expresan Cronquist y Fiszbein (2017), al enunciar que en el Perú, los estándares asumidos del Marco Común Europeo de Referencia (MCER) para la enseñanza, aprendizaje y evaluación de las lenguas, los cuales deben cumplirse para desplegar el proceso de enseñanza-aprendizaje del inglés, no son suficientes los niveles alcanzados, aunque hayan habido ciertos progresos. Esta situación ha conllevado a repensar los métodos y estrategias didácticas que se aplican, pues, estos han de estar en correspondencia con los estándares establecidos por dicho organismo, además, de asumir la enseñanza del inglés desde un enfoque didáctico basado en la enseñanza comunicativa de lenguas (EF, 2021). Pues, es de suma importancia fortalecer las destrezas orales, trascendiendo a la idea de que los estudiantes de una segunda lengua 
no solo deben aprender gramática o vocabulario, sino que su principal objetivo debe ser la capacidad comunicativa y, para eso, es imprescindible hablar (Mosquera, 2017) Lo anterior, aunado a que la realidad contextual de la pandemia causada por el Covid19, también, pone en evidencia como la enseñanza del inglés se ha visto afectada, dado que los maestros han tenido que adaptarse a otra forma de trabajo educativo, en este caso a una enseñanza virtual, conlleva a cuestionar ¿Desde cual enfoque didáctico se puede asumir el aprendizaje de la expresión oral en inglés académico? Considerando esta interrogante, me planteo como propósito presentar una revisión de la literatura que permita describir el Aprendizaje Integrado de Contenido y Lengua Extranjera (AICLE) como enfoque metodológico para el desarrollo didáctico de la enseñanza comunicativa de idiomas, en particular el inglés.

Metodológicamente el artículo de revisión deviene de un proceso de investigación teórica documental (Sánchez, 2011). En tal sentido, se busca presentar un panorama actualizado sobre el AICLE como método didáctico para el aprendizaje de la expresión oral en inglés académico. Por consiguiente, fue menester hacer una observación sistemática de la bibliografía que denota el conocimiento construido en relación al método AICLE como tema-problema de investigación.

Es de saber que el proceso de observación sistemática implicó, como expresa Estrada, (2014), el despliegue de los procedimientos metodológicos de rastreo, identificación, organización, sistematización, análisis e interpretación de datos e informaciones. Cabe resaltar que, desde lo enunciado por Mera, Barreiro, Bermúdez, Cedeño y Vera (2020), dichos procedimientos metodológicos se constituyen en la lógica de acción asumida para responder la interrogante planteada previamente como cuestionamiento guiador del proceso de investigación.

En cuanto al proceso de observación, se tiene que los libros, artículos de revistas, conferencias y publicaciones institucionales electrónicas, en idioma español y referente al periodo de veinticinco años, que comprende desde 1994-2021, se constituyeron en las unidades de observación como referentes empíricos que se utilizan para obtener los 
datos necesarios sobre el método AICLE y el conjunto de cualidades que la integran como unidad de análisis (Azcona, Manzini y Dorati, 2013).

Dichos documentos electrónicos fueron rastreados considerando algunas bases de datos científicas y de universidades como: Scielo, Redalyc, Google Academic, entre otros. Para este rastreo, se hizo uso de descriptores como termino-etiqueta de un concepto susceptible de aparecer con cierta frecuencia en los documentos indizables, y en las consultas que se realicen.

Asimismo, estos descriptores se asumieron como criterios de exploración en los motores de búsqueda en la red, para la inclusión-exclusión de documentos como unidades de observación. Atendiendo a lo planteado y considerando a Guirao-Goris, Olmedo y Ferrer (2008), se tiene que el proceso de revisión se desplego siguiendo la siguiente lógica procedimental:

- Clarificación del tema-problema de investigación

- Definición del propósito de la revisión

- Diseño de la estrategia de búsqueda y establecimiento de criterios de selección (inclusión-exclusión) de documentos.

- Ejecución de rastreo bibliográfico en las bases de datos y fuentes documentales.

- Organización sistemática e interpretación de la información

- Redacción del artículo

Finalmente, para cumplir con el propósito del presente artículo de revisión, la síntesis interpretativa sobre el Aprendizaje integrado de contenidos y lenguas extranjeras se ha estructurado en cuatro apartados, los cuales describen: primero, su origen y conceptualización; segundo, su fundamentación epistemológica; tercero, sus características básicas y, el ultimo, sus implicaciones didácticas. 


\section{DESARROLLO Y DISCUSIÓN}

\section{El "Aprendizaje integrado de contenidos y lenguas extranjeras" (AICLE/CLIL) en su origen y conceptualización}

En la actualidad, el AICLE como enfoque metodológico está cobrando gran importancia al tratar de asumir el proceso de enseñanza/aprendizaje de los idiomas de manera más innovadora y creativa, al fomentar el trabajo colaborativo y la superación de enfoques de enseñanza centrados en la clase magistral (Bote, 2016). Sin embargo, se requiere comprender ciertos conceptos, principios e implicaciones para su despliegue de manera eficaz, pues, no se puede considerar que cualquier proceso de enseñanza-aprendizaje de una lengua extranjera sea AICLE.

Cabe resaltar que AICLE (Aprendizaje integrado de contenidos y lenguas extranjeras), por lo general se le conoce a nivel mundial por sus siglas en inglés CLIL (Content Language Integrated Learning); por consiguiente, se puede asumir cualquiera de las dos denominaciones indistintamente.

Considerando a Bote (2016), se tiene que el enfoque AICLE/CLIL emerge, en 1994 como innovación curricular, de la mano de Dieter Wolff, David Marsh y Anne Maljers en la Universidad de Jyväskylä de Finlandia, escenario donde se configura contextualmente como nueva perspectiva para el aprendizaje de lenguas extranjeras (Porras, 2013 y Alfonzo, 2014). Sin embargo, siendo una innovación del continente europeo que busca fomentar el plurilingüismo en dicho continente, se pueden considerar como precedentes de este enfoque, el Conted-Based Second Language Instruction (CBI), de Estados Unido y la Enseñanza por inmersión lingüística (Inmersion Education) surgido en Canadá.

Ahora bien, para entender conceptualmente AICLE/CLIL, no solo como termino, sino también como enfoque educativo, es necesario traer a colación algunas nociones referidas a él. En tal sentido, se resalta lo referenciado por Salvador (2019), desde lo inscrito por Marsh (2012) y Mehisto et al. (2008), en relación a que AICLE/CLIL, es esencialmente una herramienta metodológica cuya esencialidad está en la integración lengua-contenido, pues, comprende una extensa gama de situaciones relacionadas con 
la experiencia de aprender materias no lingüísticas a través de un idioma extranjero como el inglés.

No obstante, Coyle et al. (2010), citados por Avilés (2020), lo define propiamente como un enfoque educativo dual, en el que se utiliza un idioma adicional para el aprendizaje y la enseñanza tanto del contenido como del idioma. Para Cendoya, Di Bin y Peluffo (2008), el AICLE/CLIL es una forma de aprendizaje que combina aspectos lingüísticos distintos de los que se usan habitualmente y áreas de conocimiento específicos del currículum, por tanto, persigue un doble objetivo. En este mismo tenor, Salvador (2019), desde lo inscrito por Eurydice (2006), expresa que es una praxis educativa que utiliza una lengua extranjera o segunda lengua para la enseñanza de asignaturas curriculares no lingüísticas.

Todas estas consideraciones hechas permiten enunciar que el AICLE/CLIL, se constituye en un enfoque metodológico guiador de una praxis educativa que se funda en la integración de los aspectos lingüísticos de un idioma de habla no habitual, con los contenidos de otras disciplinas o asignaturas curriculares comunes como Historia, Literatura, Ciencias Naturales, entre otras. Por consiguiente, procura, más que forzar la enseñanza de un idioma extranjero, desarrollar el lenguaje desde experiencias innovadoras de aprender materias no lingüísticas, a través de la adquisición de un segundo idioma.

\section{Fundamentación epistemológica del Aprendizaje integrado de contenidos y lenguas extranjeras (AICLE/CLIL)}

Para entender el devenir del enfoque AICLE/CLIL es menester ampliar la perspectiva a fin de poder hacer apropiación de su entrelazamiento con el enfoque comunicativo para la enseñanza de una lengua extranjera. Cabe decir, que este enfoque pretende el aprendizaje de otro idioma desde situaciones comunicativas reales, pues, se busca que el estudiante logre la funcionalidad efectiva del lenguaje y la capacidad comunicativa; al 
mismo tiempo que se trata de superar la centralidad en la enseñanza de la gramática/vocabulario y su corrección (Salvador, 2019).

Ahora bien, al asumir el AICLE/CLIL como metodología basada en el enfoque comunicativo para la enseñanza de otra lengua y considerando lo enunciado por Segade (2012), desde su óptica cognitivo-personalista, se puede decir que este se fundamenta epistemológicamente en la antropología de la acción personal y la lingüística cognitiva, dado que el acto comunicacional se constituye en eje de la acción humana para la construcción de conocimientos. Por consiguiente, todo acto comunicativo, que en el escenario académico implica la creación de situaciones de aprendizajes mediante tareas, actividades, juegos didácticos, entre otros, ha de manifestar tres condiciones fundamentales:

- Primero, debe existir una necesidad real de comunicar una nueva información por parte de uno de los participantes del acto comunicativo.

- Segundo, se deben considerar las opciones reales de cómo y qué se puede decir de un ente o cosa.

- Tercero y último, en todo acto comunicacional la retroalimentación o feedback es fundamental, pues, es el mecanismo que permite verificar el logro de la intención originaria del acto comunicacional mismo.

Estas tres condiciones, determinantes del acto comunicativo, en términos epistemológico, explican que toda persona aprende mediante los actos comunicativos, en relación con el otro. En un sentido antropológico, el ser humano se manifiesta con su estructura personal, pero, también se revela en la interrelación, mediante el encuentro entre yo-tu. Así, en este encuentro el lenguaje se constituye en instrumento de interacción base del acto comunicativo; entonces, el uso real del lenguaje implica contextualizarlo, es decir, escoger, en la multiplicidad de opciones conceptuales, cual es la más acertada en el contexto. Pues, esto es lo que puede conllevar a la retroalimentación como acto de negociación de significados de consolidación del acto comunicacional mismo. 
Considerando lo planteado, además de lo expresado por el mismo Segade (2012), se puede deducir, que en el proceso didáctico y de aprendizaje, la correcta estructuración de estrategias y situaciones que conlleven a la práctica continua de actos comunicacionales reales, representa una forma de desarrollar competencias lingüísticas en una segunda lengua, hasta el punto de poder lograr que los estudiantes participantes del proceso, logren capacidades y habilidades que le permiten comprender e interpretar en otro idioma, la realidad como mundo significado en toda su riqueza.

Ahora bien, desde este contexto epistemológico, el AICLE/CLIL como enfoque metodológico para la adquisición de otro idioma o lengua extranjera, pedagógicamente se fundamenta en cinco dimensiones interrelacionadas, las cuales se asumen como principios nucleares y orientadores que establecen las líneas básicas para que el docente despliegue sus prácticas educativas metodológicamente coherente y con sentido, a la vez que sirven de base para la elaboración de un currículum basado en dicho enfoque (Salvador, 2019 y Porras, 2013).

Estos cincos principios establecidos por Coyle, Hood y Marsh, los cuales se denominan las 5Cs de AICLE/CLIL, considerando a (Custodio, 2019, Salvador, 2019 y Porras, 2013), se describen de la siguiente manera:

1. Contenido. Representa el elemento vertebrador del proceso de aprendizaje, pues refiriere a la esencia básica de la asignatura curricular que acoge la aplicación del AICLE/CLIL, siendo quien orienta la planificación general a lo largo de la ruta de aprendizaje. Por consiguiente, estos han de ser creados a partir de información relevante, significativa y contextualizada; además, de ser desplegados con estrategias didácticas más eficaces, a fin de que el estudiante participe activamente en la construcción de su propio conocimiento.

2. Comunicación. También asumido como lenguaje, pues, implica el uso comunicacional que se le da a la lengua extranjera en proceso de apropiación, con las actividades de aprendizaje. En consecuencia, cuando se le da al idioma un uso 
real y más contextualizado al estudiante, este adquiere mayor significatividad y torna el aprendizaje en un proceso más motivado. Entendiendo el papel vehicular que tiene la lengua para aprender a usar la lengua misma en la interacción comunicativa, al mismo tiempo que se aprenden contenidos.

3. Cognición. En tanto que el aprendizaje implica el desarrollo de las destrezas cognitivas o procesos de pensamiento de orden superior, entonces, hacer apropiación de otro idioma o lengua extranjera desde el enfoque AICLE/CLIL, debe representar un desafío cognitivo para el estudiante, ya que solo así logrará construir sus propias interpretaciones, crear nuevos conocimientos y habilidades, a la par que desarrolla competencias comunicativas y de lenguaje.

4. Cultura. Siendo la cultura inherente al lenguaje, entonces, aprender otro idioma en el marco de AICLE/CLIL, también implica para el estudiante, ser conscientes de la realidad cultural que le rodea, además, de otras culturas. Ya que, es factor fundamental para acceder al sentido completo de la comunicación, aunado a que expone a diversos puntos de vistas y a conocimientos compartidos que elevan la conciencia del estudiante, sobre el otro y sobre sí mismo, conllevando a procesos de interculturalidad para la convivencia universal.

5. Competencias. Refiere a la capacidad de aplicar conocimientos para el abordaje y superación con éxito de situaciones concretar. Por consiguiente, desarrollar competencias lingüísticas en otro idioma desde el enfoque AICLE/CLIL, envuelve la aplicabilidad práctica de los nuevos saberes y aprendizajes logrados, tanto del contenido como de la propia lengua extranjera.

\section{Caracterización del Aprendizaje integrado de contenidos y lenguas extranjeras (AICLE/CLIL)}

En el AICLE/CLIL, como modelo metodológico para el aprendizaje que brinda oportunidades que resultan ser más realista y naturales para el desarrollo práctico de otro idioma o lengua extranjera, se pueden distinguir características que son de necesario 
conocimiento para su aplicación en contextos académicos. En tal sentido, se listan algunas de estas características del AICLE/CLIL, considerando los aportes de Cendoya, Di Bin y Peluffo (2008); Alfonso (2014); Mosquera (2017) y Salvador (2019):

1. Es una innovación curricular que se crea en virtud de favorecer a todo el estudiantado en cuanto al aprendizaje de otros idiomas o lenguas extranjeras, sin distinto de procedencia social o poder adquisitivo; por tanto, emerge vinculado a los principios de justicia social y equidad.

2. La lengua y el contenido no lingüístico se constituyen objetos de enseñanza, en igualdad de predominio, para desarrollar aptitudes lingüísticas desde los actos comunicacionales, al mismo tiempo que se genera conocimiento de contenidos y se promueven actitudes para su apropiación de manera innovadora.

3. El proceso de integración de la enseñanza de lenguas y contenido, promueve en el estudiante, el desarrollo profundo de habilidades cognitivas, convirtiéndolo en líder de su propio aprendizaje.

4. La naturalidad se constituye en la plataforma fundamental; es decir, las situaciones de aprendizaje de otro idioma o lengua extranjera, deben ser creadas de forma análogas y semejantes a las que se presentan durante el proceso natural de adquisición de la lengua materna.

5. El desarrollo de la lectura y la fluidez oral se constituyen en factores esenciales. La lectura va a permitir el logro de los objetivos tanto pedagógicos como lingüístico, por tanto, debe convertirse en un una actividad placentera y motivadora. En tanto, la fluidez oral es importante, sin embargo, esto solo se logra con la práctica y aprendiendo de los errores que se cometen, como parte natural del proceso de adquisición de la lengua.

6. Es altamente motivador, en el sentido de que la condiciones en las que se despliega el proceso de adquisición una lengua extranjera mediante su uso 
práctico y natural, produce en los estudiantes una actitud positiva hacia el aprendizaje de dicha lengua, en la medida de que estos van alcanzando logros.

7. El enfoque concreta la inclusión de estrategias y recursos innovadores, que coadyuvan a la creación de entornos de aprendizajes ricos y flexibles, con los que se construyen nuevos conocimientos pedagógicos y lingüísticos, generándose con ello, una respuesta positiva para su aplicación institucional.

Para cerrar, como puede observarse, todas estas características expuestas permiten tener una mejor comprensión de lo que significa el AICLE/CLIL, como enfoque metodológico para la adquisición de otro idioma o lengua extranjera; con la cual, se pueden desplegar mejores prácticas educativas en pro de su implementación, tal como expresa Porras (2013).

\section{El Aprendizaje integrado de contenidos y lenguas extranjeras (AICLE/CLIL) y sus implicaciones didácticas para el aprendizaje de un segundo idioma o lengua extranjera}

Los procesos y actividades de aprendizaje enfocados desde el AICLE/CLIL, para la adquisición de un segundo idioma o lengua extranjera, más allá de lograr la integración de lenguas-contenidos curriculares, lo que pretende es el desarrollo de competencias cognitivas profundas en los estudiantes, desde el sentido de la autoorganización y la autogestión de su propio conocimientos y aprendizajes.

Cabe resaltar que, para ello, es fundamental asumir las nociones de participación, interacción y colaboración como principios implicados en los procesos y actividades didácticas. Pues, como expresa Alfonso (2014) referenciando a Borrull et al. (2008), desde esta perspectiva se promueve la participación colaborativa de los estudiantes en situaciones naturales y reales de interacción comunicativa en un segundo idioma o lengua extranjera, que acontecen en un contexto de uso significativo de la lengua, ya que las condiciones se asemejan a las habituales que posibilitan su apropiación. Esto didácticamente, en palabras de Villafuerte (2019), significa que: 
Cuando los estudiantes hacen uso del discurso académico para comunicar el conocimiento que ellos poseen respecto a una materia usando el idioma extranjero, se acude al uso de la competencia de interacción. Así, los participantes trabajan juntos para negociar el tema y roles, y organizan las actividades o trayectorias a aplicar (p.41).

Ahora bien, este proceso constante de interacción comunicativa como estrategia didáctica fundamental, en la práctica se constituye en la plataforma para que el estudiante construya nuevos conocimientos de manera paulatina y a su ritmo particular, pues, se asume el aprendizaje, como un pase continuo de conciencia simple a comprensión real y, de allí, a ser competentes. En este contexto, el Lifelong Learnig Progranmme (2016), expresa que es imprescindible el proceso de andamiaje o estructuras dinámicas de apoyo, que pueden tomar diversas formas y ser dados por el docente y/o los compañeros con más experticia y en colaboración, hasta lograr la interiorización del conocimiento lingüístico y ser capaz de utilizarse y aplicarlo sin apoyo externo.

En concreto, desplegar el AICLE/CLIL como enfoque guiador de un modelo didáctico que conlleva a crear situaciones de aprendizaje donde la lengua y los contenidos de distintas asignaturas se integran, resulta altamente beneficioso, en el sentido de que imbuye al estudiante a desarrollarse en este idioma, sobre todo, a nivel comunicativo. Así, Bote (2016) y Wolff (2008), referenciado por y Lifelong Learnig Progranmme (2016), expresa que estos beneficios se traducen en un proceso de apropiación de otro idioma o lengua extranjera intricado a la interculturalidad, que conlleva a asumir los contenidos desde diversas perspectivas, trabajando las capacidades cognitivas de forma más eficaz, lo que motiva a los estudiantes a desarrollan conceptos académicos más precisos y con éxito.

\section{CONSIDERACIONES FINALES}

Atendiendo a los argumentos y explicaciones esgrimidas a lo largo del artículo, no cabe duda que el enfoque AICLE/CLIL para el aprendizaje de otro idioma o lengua extranjera, desde el punto de vista pedagógico-curricular se muestra como un paradigma 
metodológico fundamentado epistemológicamente en el acto comunicativo, donde el lenguaje se presenta como instrumento de la interacción.

En este contexto, el contenido, la comunicación, la cognición, la cultura y las competencias, como dimensiones de este enfoque, suponen un marco conceptual guiador del desarrollo de estrategias didácticas interactivas. Enfoque que ofrece la posibilidad de introducir el mundo real al contexto de una clase, transformándola en un espacio natural para hacer apropiación de contenidos temáticos y lingüísticos de manera integrada e interactiva, a la vez que se fomenta la colaboración y la interculturalidad. Por consiguiente, este proceso hace que el estudiante, al estar continuamente expuesto al uso de otra lengua en las interacciones comunicativa, se motive y desarrolle competencias lingüísticas, a la vez que mejora el pensamiento crítico y la conciencia cognitiva.

Para cerrar, mediante el enfoque AICLE/CLIL, se configura una forma de aprendizaje más efectivo para los estudiantes, pues se aboca a desarrollar competencias para el uso funcional y comunicativo de otro idioma como el inglés de manera contextualizada.

\section{FINANCIAMIENTO}

No monetario.

\section{AGRADECIMIENTO}

A la Universidad Cesar Vallejo, Chimbote; por motivar el desarrollo y fomento de la investigación.

\section{REFERENCIAS CONSULTADAS}

Alfonso, M. (2014). Definición, fundamentación y principales agentes de AICLE en la educación primaria. [Tesis de Pregrado]. Universidad Internacional de La Rioja, España. Recuperado de: https://n9.cl/os7×0 
Avilés, E. (2020). Aprendizaje integrado de contenidos y lengua extranjera. Revista de Innovación Didáctica de Madrid, 65, 09 - 18. Madrid. Recuperado de https://n9.cl/66t58

Azcona, M., Manzini, F. y Dorati, J. (2013). Precisiones metodológicas sobre la unidad de análisis y la unidad de observación. Aplicación a la investigación en Psicología. Conferencia llevada a cabo en el IV Congreso Internacional de Investigación de la Facultad de Psicología de la Universidad Nacional de La Plata. Argentina. Recuperado de: https://n9.cl/tbyx1

Bote, M. (2016). Análisis de la metodología AICLE como medio de aprendizaje de una lengua extranjera. [Tesis de Pregrado]. Universidad de Extremadura, España. Recuperado de: https://n9.cl/tbyx1

British Council (2015). Inglés en el Perú: un análisis de las políticas, las percepciones y los factores de influencia. Recuperado de https://n9.cl/uzv7e

Cendoya, A., Di Bin, V. y Peluffo, M. (2008) AICLE. Aprendizaje integrado de contenidos y lenguas extranjeras o CLIL (Content and Language Integrated Learning). Puertas Abiertas, 4(4), 65-68. En Memoria Académica. Disponible en: https://n9.cl/2fd3t

Conquistó, K., \& Fiszbein, A. (2017). El aprendizaje del inglés en América Latina [Learning English in Latin America]. Recuperado de https://n9.cl/9kj2

Education First (2021). Índice del Dominio del inglés de EF. Recuperado de https://n9.cl/vc06t

Estrada, R. (2014). Blended-learning afectivo y las herramientas interactivas de la web 3.0: Una revisión sistemática de la literatura. Revista científica electrónica de Educación y Comunicación en la Sociedad del Conocimiento, 1(14), 1695-324X. https://n9.cl/bw8gc

Fernández, R. (2021). Los idiomas con más hablantes en el mundo en 2021. Recuperado de https://n9.cl/8d5r1

García, I., Vecorena, N. y Velazco E. (2019). El nivel de inglés alcanzado en quinto grado de secundaria en tres colegios públicos de Lima metropolitana. Educación, 28(55), 80-102. DOI: https://doi.org/10.18800/educacion.201902.004 
Guairao-Goris, J., Olmedo, A \& Ferrer, E. (2008). Artículo de revisión. Revista Iberoamericana de Enfermería Comunitaria, 1(1), 6.

Lifelong Learnig Progranmme (2016). Guía CLIL. Recuperado de: https://n9.cl/atosr

Mera C., Barreiro, A., Bermúdez, R., Cedeño, B., \& Vera, D. (2020). La revisión sistemática de literatura utilizando recursos electrónicos en el desarrollo de proyectos: revisión sistemática de literatura en el desarrollo de proyectos. UNESUM-Ciencias. Revista Científica Multidisciplinaria, 4(2), 1-11. https://doi.org/10.47230/unesum-ciencias.v4.n2.2020.220

MINEDU (2015). Decreto Supremo No 012-2015-MINEDU. Recuperado de https://n9.cl/zpkmy

MINEDU (2016). Decreto Supremo $N^{\circ}$ 007-2016-MINEDU NORMAS LEGALES. Recuperado de https://n9.cl/f0d4v

Mosquera, I. (2017). La expresión oral en el aula de inglés de Primaria. Recuperado de: https://n9.cl/s5tyi

Niño-Puello, M. (2013). El inglés y su importancia en la investigación científica: algunas reflexiones. RECIA, 5(1),

243-254.

DOI: https://doi.org/10.24188/recia.v5.n1.2013.487

Peña, V. (2019). Enseñanza del inglés como lengua extranjera y desarrollo de competencias lingüísticas. [Tesis de Maestría]. Universidad Andina Simón Bolívar, Ecuador. Recuperado de https://n9.cl/ph9we

Porras, D. (2013). Análisis de CLIL (Content Language Integrated Learning) como metodología para enseñar inglés como segunda lengua. [Tesis de Pregrado]. Universidad Internacional de La Rioja, España. Recuperado de: https://n9.cl/u03yd

Salvador, C. (2019). Análisis multidimensional de la aplicación de Aprendizaje Integrado de Contenidos y Lengua en Educación Física. [Tesis Doctoral]. Universitat Jaume I, Castelló, España. Recuperado de: http://repositori.uji.es/xmlui/handle/10803/666197

Sánchez, A. (2011). Manual de redacción académica e investigativa: cómo escribir, evaluar y publicar artículos. [Archivo PDF]. Recuperado de: https://n9.cl/tls1v 
Revista Arbitrada Interdisciplinaria KOINONIA

Año VI. Vol VI. N4. Edición Especial: Educación III. 2021

Hecho el depósito de Ley: FA2016000010 ISSN: 2542-3088

FUNDACIÓN KOINONIA (F.K). Santa Ana de Coro. Venezuela.

Liliana Amalia Alegre-Alvarado

Segado Alonso, C. E. (2012). Fundamentación epistemológica del enfoque comunicativo en la enseñanza de lenguas extranjeras: una visión cognitivopersonalista. Didáctica. Lengua y Literatura, 24, 473-487. DOI: https://doi.org/10.5209/rev DIDA.2012.v24.39935

Villafuerte, J. (2019). Tecnología de la Información y Comunicación y el desarrollo de la competencia comunicativa en inglés de los futuros docentes de lengua extranjera de Ecuador: propuesta de intervención educativa. [Tesis Doctoral]. Universidad del País Vasco, España. Recuperado de: https://addi.ehu.es/handle/10810/35071

(O2021 por los autores. Este artículo es de acceso abierto y distribuido según los términos y condiciones de la licencia Creative Commons Atribución-NoComercial-Compartirlgual 4.0 Internacional (CC BY-NC-SA 4.0)

(https://creativecommons.org/licenses/by-nc-sa/4.0/). 